\title{
Bending Stiffness Theoretical and Experimental Analysis of Triangular Prism Mast with Tape-Spring hyperelastic hinges
}

\author{
YANG Hui ${ }^{1, a}$, DONG Fei ${ }^{1, b}$ and Lu Siliang ${ }^{1, c}$ \\ ${ }^{1}$ College of electrical Engineering and Automation, Anhui University, China \\ ahuiyang_0431@163.com, ${ }^{\mathrm{b}}$ lusliang@mail.ustc.edu.cn, ${ }^{\mathrm{c}}$ 2506020201@163.com
}

\begin{abstract}
Keywords: Deployable mechanism; triangular prism mast, tape-spring hyperelastic hinges; bending stiffness; experimental analysis.

Abstract. This study reports on the design, analysis and testing of a new ten-module triangular prism mast. The triangular prism mast is designed such that it is derived by tape-spring hyperelastic hinges using the stored strain energy. Static bending and theoretical models are established to predict the triangular prism mast properties. Mechanical tests of a two modules triangular prism mast were conducted to measure these properties as well. The bending stiffness test results were shown a $-9.41 \%$ difference.
\end{abstract}

\section{Introduction}

Flexible hinges, which are folded elastically can self-deploy by releasing stored strain energy, which consist of a fewer component parts, can be manufactured conveniently[1,2]. Flexible hinges have several advantages for space applications, including a low mass-to-deployed-stiffness ratio, cost, and self-latch[3].

With the increasing demand, flexible hinges have been widely used as folding and deployment mechanisms in deployable structures, such as synthetic aperture radars (SARs), solar arrays and antenna booms. Tape-spring hinges have been used in the Japanese Mars orbiter PLANT-B for bar-like deployment structures of the thermal plasma analyzer[4,5,6,7]. Howerever, there are still some engineering problem for the tape-spring hyperelastic hinge applying to a deployable mechanism.

This paper proposed a new ten-unit triangular prism mast with tape-spring hyperelastic hinges. Static bending stiffness and compressed stiffness theoretical models are established. The tests are performed to verify the theoretical models of the triangular prism mast.

\section{Design and static stiffness analysis}

Structures design. A new ten-module triangular prism mast with tape-spring hinges is shown in Fig. 1. Adjacent two units are connected by triangular frame. The length of triangular frame is $469 \mathrm{~mm}$, stowed and deployed longitudinal length of ten units prism mast are $475.2 \mathrm{~mm}, 5278 \mathrm{~mm}$ respectively. All components except standard parts are manufactured by duralumin $2 \mathrm{~A} 12$.

Each modulus triangular prism mast with tape-spring hyperelastic hinge contains three longitudinal links and two triangular frames. The tape-spring hyperelastic hinge are used to drive and lock triangular prism mast. Lateral area of each modulus has a series of flexible kevlar rope with tensile force $30 \mathrm{~N}$ which ensures high stiffness of the deployed prism mast and reduce deploying impact. There is one resin-based carbon fiber drive pipe one each end of the tensile lock which prevent involvement during deploying process.

The tape-spring hyperelastic hinge folded with $180^{\circ}$, the two triangular frame close to each other, and three longitudinal links folded between the two triangular frames when the mast stowed. The tape-spring drive the mast to deploy. After deploying, the tape-spring hyperelastic hinge restore original shape, the Kevlar ropes tensile and the triangular prism mast was rigidified to a structure. 


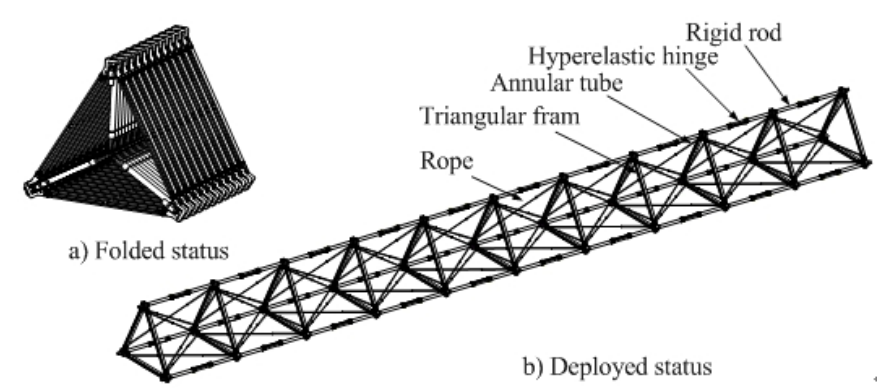

Fig. 1. Ten units of triangular prism mast

Static bending analysis. The triangular prism mast main bear bending moment under working state. It is necessary to analyze the bending stiffness. Axial force $\mathrm{F}$ is applied on the point $\mathrm{A}$ of the cross section, and axial force $-\mathrm{F}$ is applied on both point $\mathrm{B}$ and $\mathrm{C}$. Circum-circle radius of cross section is $R_{1}=l_{b} / \sqrt{3}$. Bending force diagram is shown as figure 2, and spring-damp system is stranded for the tape-spring hyperelastic hinge.

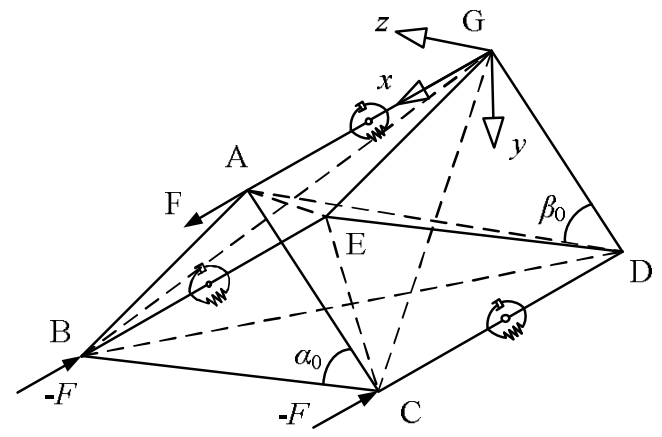

Fig. 2. Bending force diagram

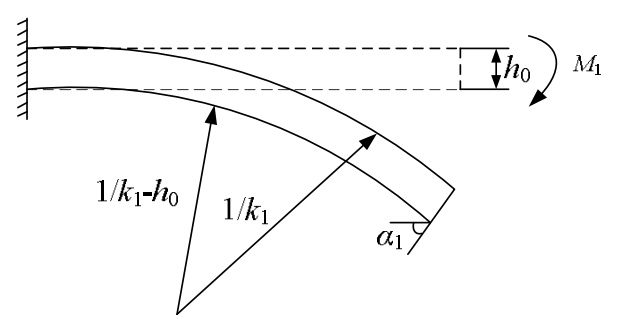

Fig. 3. Bend schematic diagram

The forces are asymmetrical about $x y$ plane because the cross section is equilateral triangle. The equations of point $\mathrm{A}$ and point $\mathrm{B}$ for this case are as follows :

$$
\begin{gathered}
2 F_{A D} \cos \beta_{0} \cos \frac{\alpha_{0}}{2}-2 F_{b} \cos \frac{\alpha_{0}}{2}=0 \\
F_{0}-F_{A D} \sin \beta_{0}+F_{A G}=0 \\
F_{b} \cos \frac{\alpha_{0}}{2}-F_{B G} \cos \beta_{0} \cos \frac{\alpha_{0}}{2}=0 \\
-F_{b} \sin \frac{\alpha_{0}}{2}+F_{B G} \cos \beta_{0} \sin \frac{\alpha_{0}}{2}+F_{B D} \cos \beta_{0}-F_{b}=0 \\
F_{B E}-F_{0}-F_{B D} \sin \beta_{0}-F_{B G} \sin \beta_{0}=0
\end{gathered}
$$

Where $F_{A D} 、 F_{B G} 、 F_{B D}$ are the forces of the rods, link $\mathrm{AD}$, link BG and link BD, respectively; $F_{A G}$ 、 $F_{B E}$ are the forces of both end of longitudinal links; $F_{b}$ is the force of cross link; $\alpha_{0}$ is angle between two adjacent cross links, $\alpha_{0}=\pi / 3 ; \beta_{0}$ is the angle between rod and cross link.

The equivalent moment $M_{1}$ applied on the end is

$$
M_{1}=\left(F_{A G}+F_{B E}\right) \cdot \frac{l_{b}}{\sqrt{3}}
$$

Where $l_{b}$ is the cross link length.

Simultaneous equation (1a) to (2c), we get

$$
\begin{gathered}
F_{A D}=F_{B G}=F_{B D}=F_{b} / \cos \beta_{0}=F_{r o} \\
F_{A G}=2 F_{r o} \sin \beta_{0}-F_{0} \\
F_{B E}=2 F_{r o} \sin \beta_{0}+F_{0}
\end{gathered}
$$


Where $F_{r o}$ is rod pre-tension force.

Compared equations (4), it is found that rope tension forces are equaled with each other and the tension force of the upper longitudinal link is lower than the compress force of the bottom longitudinal link. Figure 3 is bend schematic diagram of the triangular prism mast under pure bending moment $M_{1}$. The upper and lower arc of the triangular prism mast under bending have following relation equation.

$$
\begin{gathered}
\frac{1}{k_{1}} \cdot \alpha_{1}=n_{2} \cdot l_{m}+\delta_{u} \\
\left(\frac{1}{k_{1}}-h_{0}\right) \cdot \alpha_{1}=n_{2} \cdot l_{m}-\delta_{b}
\end{gathered}
$$

Where $k_{1}$ is upper arc curvature, $\alpha_{1}$ is bending angle, $n_{2}$ is the modulus number $\left(n_{2}=1,2,3, \ldots\right), l_{m}$ is longitudinal length of each triangular prism mast modulus, $\delta_{u}$ is the total tensile deformation of the upper longitudinal link, $h_{0}$ is projection height of cross section on $x y$ plane, $h_{0}=\frac{\sqrt{3}}{2} \cdot l_{t}, \delta_{b}$ is the total compressed deformation of the lower longitudinal link.

When the triangular prism mast is bended, the longitudinal tensile deformation and compressed deformation are the total deformation of the tape-spring hyperelastic hinge and the short longitudinal link.

$$
\begin{aligned}
& \delta_{u}=n_{2} \cdot F_{A G} \cdot\left(\frac{2 l_{1}}{E_{1} A_{1}}+\frac{l_{2}}{n_{1} a_{11}}\right) \\
& \delta_{b}=n_{2} \cdot F_{B E} \cdot\left(\frac{2 l_{1}}{E_{1} A_{1}}+\frac{l_{2}}{n_{1} a_{11}}\right)
\end{aligned}
$$

Where $n_{1}$-is the number of tape-spring in the hyperelastic hinge $n_{1}=2,4,6, \ldots, l_{1}$ is longitudinal link length, $l_{2}$ is the tape-spring hyperelastic hinge length, $A_{1}$ is cross section area of the longitudinal rigid link, $a_{11}$ is tensile stiffness per radian of the tape-spring hyperelastic hinge, $a_{11}=\frac{E t}{1-v^{2}}, E_{1}$ is Young modulus of the short longitudinal link.

Simultaneous equation (5) to (6), the bending angle of the triangular prism mast is as follows

$$
\alpha_{1}=\frac{2 n_{2}}{\sqrt{3} l_{b}} \cdot 4 F_{r o} \sin \beta_{0} \cdot\left(\frac{2 l_{1}}{E_{1} A_{1}}+\frac{l_{2}}{n_{1} a_{11}}\right)
$$

Neutral surface keeps the original length when the triangular prism mast is bending. Thus, the neutral surface bending curvature $k_{1}$ is gotten.

$$
k_{1}=\frac{\alpha_{1}}{n_{2} l_{m}}
$$

The triangular prism mast integral pure bend is similar to the pure bending of beam. According to beam pure bending theory, the upper longitudinal bending curvature $k_{1}$ is as follows.

$$
k_{1}=\frac{M_{1}}{E I}
$$

Where $E I$ - is the cross section bending stiffness of the triangular prism mast.

Simultaneous equation (3), (4), (6) to (9), the bending stiffness of the triangular prism mast is gotten.

$$
E I=\frac{l_{b}^{2} \cdot\left(2 l_{1}+l_{2}\right)}{2\left(\frac{2 l_{1}}{E_{1} A_{1}}+\frac{l_{2}\left(1-v^{2}\right)}{n_{1} E t}\right)}
$$

Where $l_{b}$ is the cross link length, and $E$ is Young modulus of the tape-spring hyperelastic hinge. 
Bending stiffness of the triangular prism mast has relation with the cross link length, the longitudinal rigid link length, the length and the Young modulus of the tape-spring hyperelastic hinge, and has no relation with the tensile stiffness of the ropes and the cross link.

\section{Experimental investigation}

Two units triangular prism mast. To validate the precision of static stiffness theoretical model, two units triangular prism mast is developed. Figure 4 is folding and deployment configurations of triangular prism mast with hyperelastic hinges.

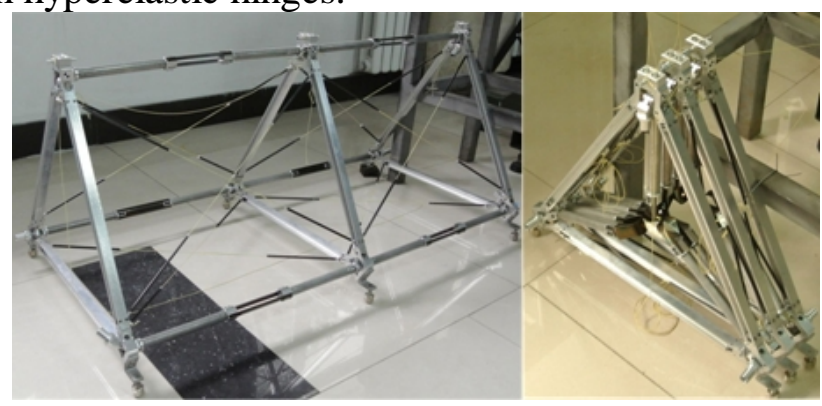
a) Deployed status
b) Folded status

Fig. 4. Folding and deployment configuration of triangular prism mast with hyperelastic hinges

Two adjacent units triangular prism mast are closed to each other by locating pins and fastened to a work holder by a rope when it is folded. At this time, the longitudinal links are stowed into the prism frames and tension ropes are located to grooves. After releasing the tension ropes, the triangular prism mast is deploying by the driving of the tape-spring hyperelastic hinges. Spherical wheels support the mast to reduce the influence of gravity.

Bending stiffness test. Bending stiffness test apparatus is shown in Figure 5. One end is fixed on the optical precision platform, the other end is suspend. The weights are applied through a crown block and the weight is added $2 \mathrm{~kg}$ every time. A laser displacement sensor is used to capture the tip displacement of the triangular prism mast. A data acquisition instrument is applied to collect and record data. Bend load and end displacement for the two modulus triangular prism mast is listed in Table 1.

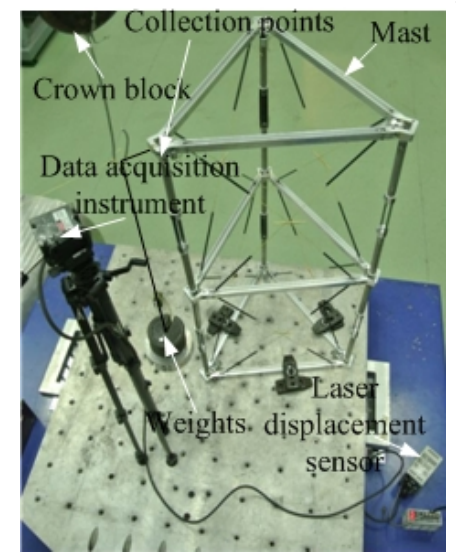

Fig. 5. Bending stiffness test apparatus

Table. 1. Bend load and end displacement for the two modulus triangular prism mast

\begin{tabular}{ccccc}
\hline Load $/ \mathrm{N}$ & 20 & 40 & 60 & 80 \\
\hline Deformation $/ \mathrm{mm}$ & 0.639 & 1.497 & 2.598 & 3.6289
\end{tabular}

According to mechanics of materials, when a cantilever is applied concentrated force load on impending end, deformation is as follows :

$$
w_{B}=\frac{F_{e} l_{t}^{3}}{3 E I}
$$


Where $w_{B}$-the deformation of the impending end, $F_{e}$ - concentrated force load, $l_{t}$ - total longitudinal length of the triangular prism mast.

The bending stiffness $E I$ of the triangular prism mast is derived as follows:

$$
E I=\frac{F_{e} l_{t}^{3}}{3 w_{B}}
$$

According to the bending load and the deformation of the impending end at table 1, the experimental bending stiffness of the triangular prism mast is $10086.203 \mathrm{Nm}^{2}$ according to the equation (22). and the theoretical bending stiffness is $11035.792 \mathrm{Nm}^{2}$ according to the equation (10). The relative error between the theoretical and experimental results is $-9.41 \%$ which validate the precision of the theoretical model.

\section{Conclusions}

Ten units triangular prism mast with tape-spring hyperelastic hinge is designed. The two units triangular prism mast is developed to investigate the mechanical characteristics through theoretical and experimental methods. The main contents are as follows :

(1)The theoretical bending stiffness model of the triangular prism mast is derived by statics method. (2) The two units triangular prism mast is developed to test the bending stiffness. The relative error between the theoretical and experimental results is $-9.41 \%$. The test results validate the precision of the theoretical model.

\section{Acknowledgements}

This project was supported by the College Discipline Innovation Wisdom Plan in China (Grant No. B07018), the Excellent Engineers Projects of Mechanism design,manufacturing and automatization(No.J10118417003).

\section{References}

[1] K.A. Seffen: J. Applied. Mech.-T. ASME, Vol. 68 (2001), p. 369-375.

[2] J. Block, M. Straubel, M. Acta. Astronaut, Vol. 68 (2011), 7-8, p. 984-992.

[3] M. Mobrem, D.S. Adams. J. Spacecraft. Rockets Vol. 46 (2009), 2, p. 394-402.

[4] K. Oya, J. Onoda. . 43rd AIAA/ASME/ASCE/AHS /ASC structures, structural dynamics, and materials conference 22-25 April 2002, Denver, Colorado(2002)

[5] H.M.Y.C. Mallikarachchi, S. Pellegrino. J. Spacecraft. Rockets. Vol.48(2011), 1, p. 187-198.

[6] H. Yang, Z.Q. Deng, R.Q. Liu, Y. Wang, H.W. Guo. Chin. J. Mech. Eng.-En, Vol.27 (2014), 2, p.279-286.

[7] H. Yang, R.Q. Liu, Y. Wang, Z.Q. Deng, H.W. Guo. Structural and Multidisciplinary Optimization, Vol. 51 (2015), 6, p. 1373-1384. 